\title{
Authors' Reply to Letter: Role of Contrast-Enhanced Ultrasound (CEUS) in Paediatric Practice: An EFSUMB Position Statement
}

We thank the author for the interest in recent statement on use of contrast-enhanced ultrasound (CEUS) in the paediatric population [1], and note the number of concerns raised. The author makes numerous valid points with regards to the use of CEUS in the paediatric patient and highlights, quite correctly, the large number of limitations of CEUS. We believe the point of the position statement has been "missed"; CEUS will not replace CT and MR imaging but should be seen as an additional, problem solving or alternative child friendly diagnostic tool that is safe and easily repeatable in a clinical setting.

The authors do not claim to be able to considerably reduce $\mathrm{CT}$ and MR imaging in clinical practice but advocate that there is potential for reduction with the use of CEUS. There will always be the need for CT and MR imaging, but very often, and this is true both in the adult and paediatric population, physicians and radiologists are all too eager to "press-the-button" to invoke the use of more expensive and often harmful imaging prior to exhausting the potential of the least harmful imaging modality, i. e. ultrasound. Of course assessment for staging of malignant disease will need CT imaging, but is CT imaging really needed when an incidental focal lesion is present on a baseline ultrasound [2]? Is CT follow-up really necessary when there is an isolated spleen injury following blunt abdominal trauma [3]?

The ability to characterise a liver lesion immediately and accurately reduces parent anxiety particularly when the likelihood of malignancy is low. No doubt the addition of CEUS to an ultrasound examination requires valuable physician or radiologist time, but reduces potential patient morbidity considerably; the patient, in this case the child, is paying for the idleness of the physician or radiologist. It is far too easy to "order" a CT or MR examination, and report remotely at physician or radiologist convenience. Our statement is designed to encourage the paediatric imaging community to move forward and embrace this technique for the benefit of their clients, and highlights areas to consider where CEUS usefulness may be further explored.

It is important to appreciate that the statement follows the introduction of SonoVue ${ }^{\mathrm{TM}} /$ Lumason $^{\mathrm{TM}}$ (Bracco, SpA, Milan) for liver application in the paediatric population in the United States of America, licenced for use by the Food and Drug Administration (FDA) following approval without any prior conducted dedicated clinical trial. The reason for this is undoubtedly the need to reduce the medical radiation burden carried by the American child, carried into adulthood increasing the risk of later life cancer, based on real data [4].

The actuality of a CEUS examination is that licencing was restricted to adults (until recent FDA approval) for intravenous use, and then only to the heart, liver, breast and peripheral vessels. This has not hampered the adult physician and radiologist from dutifully exploring many other areas, and generally establishing the potency of CEUS in the diagnosis and management of many disease processes with numerous publications in the literature, summarised in EFSUMB guidelines [5]. Without this pioneering work by numerous practitioners in Europe the current status of CEUS would not be acknowledged as significant. This is pertinent to the point raised by the author with regards to the statement that conclusions are from a radiological culture based on adults. The many authors of the EFSUMB statement are drawn from a variety of backgrounds, all with extensive experience of CEUS both in adults and children, and many are paediatric practitioners; all driven by their vision of improving imaging care for the child using CEUS. Without the skill and observations of this group and many other unheralded practitioners across Europe, this position statement would not have been possible. The lead needs to be taken by paediatric practitioners, who to date, are unfortunately few in number.

We believe the problem lies elsewhere; there is no ideal imaging method for children and any addition that resolves a problem should be welcomed. However, the rigid implementation of CT and MR imaging in many clinical protocols precludes the introduction of CEUS as a problem solving tool. This is clearly highlighted by the author's statement that the recent guidelines issued by ESGAR/ESPR [6], excludes the use of CEUS in inflammatory bowel disease in children; has this been extensively investigated? The probability is that it has not and without encouragement will never be, driven by vested interests in existing techniques to the detriment of the child. Extensive literature exists in the adult [7] and this should be extended to the child; surely a rapid, safe, cost-effective and child friendly examination is a mandatory requirement in this young population with a potential lifelong disease.

We hope this reply as well as the position statement will overcome reactionary attitudes to the more widespread use of CEUS in children and allow for the safe care of all our children.

\section{The Authors}

Paul S. Sidhu' ${ }^{1}$, Vito Cantisani²,

Annamaria Deganello ${ }^{1}$, Christoph F. Dietrich ${ }^{3}$, C. Duran ${ }^{4}$, Doris Franke ${ }^{5}$, Zoltan Harkanyi ${ }^{6}$, Wojciech Kosiak ${ }^{7}$, Vittorio Miele ${ }^{8}$, Alkaterini Ntoulia', Maciej Piskunowicz ${ }^{9}$, Maria Sellars ${ }^{1}$, Odd Helge Gilja ${ }^{10}$

\footnotetext{
1 Department of Radiology, King's College London, United Kingdom

${ }^{2}$ Department of Radiology, "Sapienza" University of Rome, Rome, Italy

${ }^{3}$ Innere Medizin 2, Caritas-Krankenhaus, Bad Mergentheim, Germany

${ }^{4}$ Diagnostic Imaging, UDIAT-CD. CSPT., Sabadell, Spain

${ }^{5}$ Department of Pediatric Kidney, Liver and Metabolic Diseases, MHH, Hannover, Germany

${ }^{6}$ Radiology, Heim Pal Gyermekkorhaz, Budapest, Hungary

${ }^{7}$ Department of Paediatric, Haematology, Oncology and Endocrinology, Medical University of Gdansk, Poland

${ }^{8}$ Emergency Radiology, S. Camillo Hospital, Rome, Italy

${ }^{9}$ Radiology, Medical University of Gdansk, Poland
} 
${ }^{10}$ Department of Clinical Medicine, Universitetet $i$ Bergen Det medisinsk-odontologiske fakultet, Bergen, Norway

\section{Correspondence}

\section{Dr. Paul S Sidhu}

Department of Radiology, King's College London

King's College Hospital

Denmark Hill

SE5 9RS London

United Kingdom

paulsidhu@nhs.net

\section{References}

[1] Sidhu PS, Cantisani V, Deganello A et al. Role of contrast-enhanced ultrasound (CEUS) in paediatric practice: An EFSUMB position statement. Ultraschall in Med 2017; 1: 1

[2] Jacob J, Deganello A, Sellars ME et al. Contrast Enhanced Ultrasound (CEUS) Characterization of Grey-scale Sonographic Indeterminate Focal Liver Lesions in Paediatric Practice. Ultraschall in Med 2013; 34: 529540

[3] Durkin N, Deganello A, Sellars ME et al. Posttraumatic liver and spleen pseudoaneurysms in children: diagnosis, management and follow-up screening using contrast enhanced ultrasound (CEUS). J Pediat Surg 2016; 51: 289-292

[4] Pearce MS, Salotti JA, Little MP et al. Radiation exposure from CT scans in childhood and subsequent risk of leukaemia and brain tumours: a retrospective cohort study. Lancet 2012; 380: 499-505

[5] Taylor SA, Avni F, Cronin C et al. The first joint ESGAR/ ESPR consensus statement on the technical performance of cross-sectional small bowel and colonic imaging. Eur Radiol 2016; In press

[6] Piscaglia F, Nolsoe C, Dietrich C et al. The EFSUMB guidelines and recommendations on the clinical practice of contrast enhanced ultrasound (CEUS): update 2011 on non-hepatic applications. Ultraschall in Med 2012; 32: 33-59

[7] Medellin-Kowalewski A, Wilkens R, Wilson A et al. Quantitative Contrast-Enhanced Ultrasound Parameters in Crohn Disease: Their Role in Disease Activity Determination With Ultrasound. Am J Roentgenol 2015; 206 : $64-73$

\section{Bibliography}

DOI http://dx.doi.org/10.1055/s-0043-106461

Published online: 2017 | Ultraschall in Med 2017; 38: 446-449 @ Georg Thieme Verlag KG Stuttgart - New York

ISSN 0172-4614 